\title{
"Depresión e ideación suicida en los adolescentes de una Institución Educativa Pública del distrito de Chongos Bajo - Perú 2014"
}

\author{
"Depression and suicidal ideation in adolescents from a Public Educational Institution district Chongos \\ Bajo - Perú 2014" \\ Nesil Flores Palian ${ }^{1}$, Yesenia Perales Rojas², Walter Gómez Gonzales ${ }^{3}$
}

\section{RESUMEN}

Objetivo: Determinar la relación que existe entre depresión e ideación suicida en los adolescentes de una Institución Educativa Publica del Distrito de Chongos Bajo-Chupaca 2014. Materiales y Métodos: El tipo de investigación utilizada fue el cuantitativo, el diseño de la investigación fue un estudio no experimental de naturaleza transversal, prospectivo y descriptivo correlacional. La población en estudio estuvo constituida por 260 adolescentes, siendo la muestra de 156 estudiantes de la Institución Educativa "Santiago León" del Distrito de Chongos Bajo. Se aplicó la Escala de valoración de Hamilton para evaluar Depresión y la Escala de Ideación Suicida de Beck (ISB) para evaluar Ideación Suicida. Para el procesamiento de los datos se utilizó el software estadístico SPSS versión 21.0 para Windows y para el análisis de los datos y medir el nivel de correlación de las variables en estudio, la Prueba de "r" de Pearson, aceptándose como significativo valores p $<0.05$. Resultados: Los productos de las correlaciones nos muestra que los diferentes niveles de depresión e Ideación Suicida de los adolescentes están relacionados siendo su coeficiente de correlación 0,560 significativa al nivel 0,01 (2 Colas/ Bilateral), sin embargo el que tiene la mayor fuerza de correlación, es el Nivel de Depresión Muy Severa con el Nivel de Ideación Suicida Grave, siendo su coeficiente de correlación 0,829 significativa al nivel 0,01 (2 Colas/ Bilateral). Conclusión: Depresión e Ideación Suicida, están relacionados en los adolescentes encuestados de la Institución Educativa "Santiago León” del Distrito de Chongos Bajo en el año 2014, siendo su coeficiente de correlación 0,560 significativa al nivel 0,01 (2 Colas/ Bilateral). El Nivel de Depresión Muy Severa y el Nivel de Ideación Suicida Grave, tienen la mayor fuerza de correlación, siendo su coeficiente de correlación 0,829 significativa al nivel 0,01 (2 Colas/ Bilateral). Es necesario, importante e ineludible la implementación de programas preventivos de la depresión e ideación suicida y de intervención en favor de los estudiantes de esta Institución Educativa y de otras de escenarios socio geográficos similares, siendo una responsabilidad moral y científica de ocuparse no sólo de instruir a sus estudiantes.

Palabras Clave: Depresión - Ideación suicida- Adolescentes.

\begin{abstract}
Objective: To determine the relationship between depression and suicidal ideation in adolescents of Secondary School Public of Chongos Bajo's District -Chupaca 2014. Materials and Methods: The research used was quantitative, the research design was a non-experimental study of nature cross-sectional prospective and descriptive correlational. The study population consisted of 260 adolescents, using a sample of 156 students of "Santiago Leon" Secondary School of Chongos Bajo District. Was applied the Hamilton Rating scale for to assess Depression and the Suicidal Ideation Scale Beck (ISB) to assess Suicidal Ideation. For data processing was used the statistical software SPSS version 21.0 for Windows and for data analysis and measure the level of correlation of these study variables, the Pearson's "r" test accepted as significant values $\mathrm{p}<0.05$. Results: The products of correlations, shows that different Levels of Depression and Suicidal Ideation in adolescents are related, the correlation coefficient was 0.560 significant at the 0.01 level (2 tailed / Bilateral), however the one who has the strongest correlation is the Level of Severe Depression with the Level of Serious Suicidal Ideation and correlation coefficient 0.829 significant at the 0.01 level (2 tailed/ Bilateral).Conclusion: Depression and Suicidal Ideation are related in adolescents surveyed of "Santiago Leon" Secondary School of Chongos Bajo District in 2014, the correlation coefficient was 0.560 significant at the 0.01 level (2 tailed / Bilateral). The Level of Severe Depression and the Level of Serious Suicidal Ideation have the strongest correlation and correlation coefficient 0.829 significant at the 0.01 level ( 2 tailed / Bilateral). Is necessary, important and unavoidable the implementation of prevention programs for
\end{abstract}


depression and suicidal ideation, besides of that the intervention for students of this educational institution and others similar Socio-geographic scenarios, being a moral and scientific responsibility to take care not only in instruct their students.

Key words: Depression - Suicidal ideation- Adolescents

${ }^{1}$ Nesil Flores Palian, Universidad Nacional del Centro del Perú.

2 Yesenia Perales Rojas, Universidad Nacional del Centro del Perú

${ }^{3}$ Walter Gómez Gonzales, Doctor en Ciencias de la Salud, Universidad Norbert Wiener.

\section{INTRODUCCIÓN}

Actualmente la Depresión y el Suicidio se han convertido en un problema de Salud Pública a nivel Mundial debido a su incremento paulatino en los últimos años. En especial, en grupos de población de alto riesgo como son los adolescentes y jóvenes.

La Organización Mundial de la Salud (1) afirma que para el año 2020, la depresión será la segunda causa de incapacidad del mundo, lo que pone a este trastorno anímico y mental en un lugar bastante preocupante, más si se considera que las consecuencias de sufrirla se hacen presente no solo en la vida personal y profesional, sino que también, en algunos casos, puede terminar en tragedias mayores si se le permite prosperar.

Asimismo el suicidio se encuentra entre las 3 primeras causas de muerte en las personas de 15 a 44 años y segunda causa de muerte en el grupo de 10 a 24 años. Las estimaciones de la Organización Mundial de la Salud (2) para el año 2020 indican que las defunciones por suicidio aumentarán un $50 \%$, alcanzando 1.5 millones de muertes anuales a nivel mundial.El interés de esta investigación, es estudiar los niveles de depresión y los niveles de ideación suicida en los adolescentes. Para ello el

\section{MATERIALES Y MÉTODOS}

\section{Tipo de investigación:}

Por el tipo de investigación es descriptivo, por el tiempo de recolección de datos es retrospectivo, por el tipo de intervención del investigador es observacional.

\section{Diseño de investigación:}

El diseño de investigación es no experimental correlacional, de naturaleza transversal ya que se observará situaciones ya existentes, no provocadas en la investigación. objetivo general que se propone es determinar la relación que existe entre depresión e ideación suicida en los adolescentes de la Institución Educativa "Santiago León" del Distrito de Chongos Bajo-Chupaca 2014. La hipótesis que se plantea es: Existe relación significativa entre depresión e ideación suicida en los adolescentes de la Institución Educativa "Santiago León" del Distrito de Chongos Bajo-Chupaca 2014. Cabe resaltar que esta investigación no solo se dedica al análisis de datos recolectados, sino que también busca dar la solución al problema. En la actualidad no existe un estudio epidemiológico sobre depresión e ideación suicida en los adolescentes de la Institución Educativa "Santiago León" de Chongos Bajo- Chupaca, es por ello la iniciativa a realizar dicha investigación, debido a la incidencia de casos presentados tanto en Depresión como en el suicidio y para ello se plantea el estudio desde la perspectiva Preventivo-Promocional, ya que la Enfermera como profesional de la Salud vinculado a la Salud Pública y Comunitaria, promueve el cuidado de las personas, más aún en etapas de vida vulnerables como la adolescencia, es por ello que se considera necesario la realización de esta investigación.

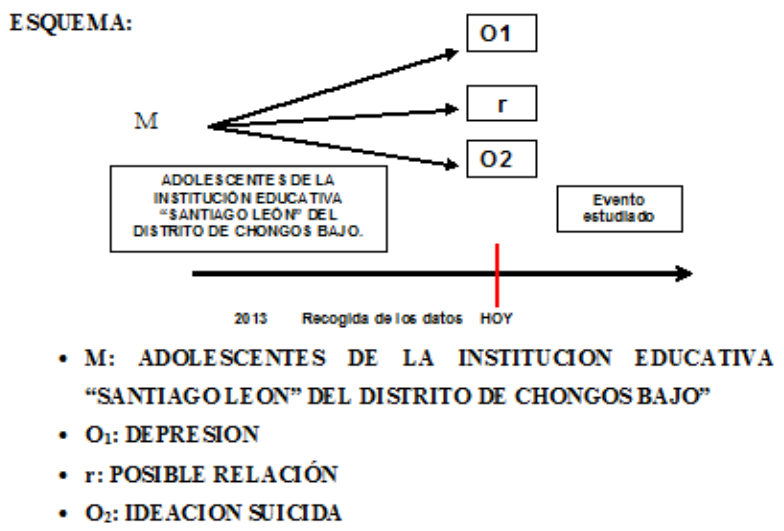


Ámbito de estudio:

Para la investigación se tomó como área de estudio a la Institución Educativa "Santiago León" del Distrito de Chongos Bajo. Ubicado en el Departamento de Junín, Provincia de Chupaca, Distrito de Chongos Bajo a $3275 \mathrm{msnm}$ y a $22 \mathrm{~km}$ de la Provincia de Huancayo.

\section{Población y muestra:}

La presente investigación utilizo como población total a $\mathbf{2 6 0}$ adolescentes de la Institución Educativa "Santiago León" del Distrito de Chongos Bajo. Matriculados en el año 2014, datos que fueron proporcionados por la Institución Educativa "Santiago León".

\section{Muestra:}

La muestra estuvo constituida por los adolescentes de la Institución Educativa "Santiago León” del Distrito de Chongos Bajo. Se seleccionó una muestra probabilística, de las cuales se tomaron a 156 adolescentes;

Para calcular el tamaño de la muestra se utilizó la siguiente fórmula:

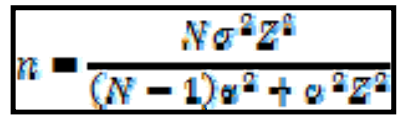

Donde:

$\mathrm{n}=$ Tamaño de la muestra

$\mathrm{N}=$ Tamaño de la población.

$\sigma=$ Desviación estándar de la población

$Z=$ Valor obtenido mediante niveles de confianza.

$\mathrm{e}=$ Límite aceptable de error muestral.

Se tomó del Primer Grado (10 estudiantes), del Segundo Grado (59 estudiantes), del Cuarto Grado (40 estudiantes), y del Quinto Grado (47 estudiantes), entre varones y mujeres. No se tomó del Tercer Grado ya que dicha población fue tomada como Prueba Piloto.

\section{Técnicas e instrumentos de recolección de datos:}

Para la recolección de los datos de las variables se empleó la técnica de la encuesta, utilizando como instrumentos:

\section{Primer Instrumento:}

\section{Escala de la Depresión de Hamilton:}

Dicho instrumento es aplicado en el Perú por el Ministerio de Salud, se difundió a todos los niveles del sector salud a través de la guía de práctica clínica en Salud Mental y Psiquiatría por la Resolución Ministerial n 422-2005/MINSA. El $1^{\circ}$
De Junio del 2005 por el entonces Dr. Hernán Garrido Lecca Montañes Ministro de Salud.

La escala de valoración de Hamilton es una escala, heteroaplicable, su versión original constaba de 21 ítems, posteriormente se realizó una versión reducida con 17 ítems, que es validado por el Ministerio de Salud.

Cada pregunta tiene entre tres y cinco posibles respuestas, con una puntuación de 0-2 ó de 0-4 respectivamente. La puntuación total va de 0 a 52 . Pueden usarse diferentes puntos de corte a la hora de clasificar el cuadro depresivo. MINSA, recomienda emplear los siguientes puntos de corte: No Deprimido: 0-7, Depresión Ligera/Menor: 813, Depresión Moderada: 14-18, Depresión Severa: 19-22, Depresión Muy Severa: >23.

\section{Segundo Instrumento:}

\section{Escala de Ideación Suicida de Aaron Beck:}

Dicho instrumento también es aplicable y validado en el Perú por el Ministerio de Salud en las guías de práctica clínica en Salud Mental y Psiquiatría. Esta escala fue diseñada para cuantificar y evaluar la recurrencia consistente de pensamientos suicidas; poner en la balanza varias dimensiones de auto destructividad y pensamientos o deseos.

La consta de 19 reactivos aplicados en el contexto de una entrevista auto administrada cuyos reactivos miden la intensidad de las actitudes, conductas y planes específicos para suicidarse. Cada reactivo se registró en un formato de respuesta de tres opciones con un rango de 0 a 2; las dimensiones se sumaron para obtener un puntaje global, de este modo el rango teórico del puntaje es de 0-38 puntos, así mismo se categorizo las puntuaciones en las áreas: Nulo/Leve: 0-9 puntos, Moderado: 1018 puntos, Agudo: $19-28$ puntos y Grave de 29-38 puntos.

\section{Procedimientos para la recolección de datos:}

La recolección de datos se realizó mediante las coordinaciones pertinentes con las autoridades de las Institución Educativa "Santiago León" del Distrito de Chongos Bajo-Chupaca, donde se llevó a cabo la investigación. Los datos fueron recolectados de ambas variables en una sola persona, en un solo momento del Mes de Diciembre. Durante 20 a 30 minutos aproximadamente en los horarios de tutoría; contando con la participación de todos los adolescentes que dieron su consentimiento para ser partícipes de la investigación. 


\section{Técnicas de procesamiento y análisis de datos:}

Primero se conformó una base de datos en el programa Microsoft Office Excel 2013 para un análisis descriptivo y tablas de frecuencia de las variables. Después para el análisis estadístico se empleó el programa IBM SPSS Statistics versión 21 , mediante este programa se pasó a realizar el análisis del coeficiente de correlación de los puntajes de la Depresión y la Ideación Suicida en los adolescentes de la Institución Educativa

\section{RESULTADOS}

La presentación de los resultados de la investigación por el tipo de diseño metodológico descriptivo/ correlacional y la búsqueda de la fuerza de relaciones entre la variable Depresión e Ideación suicida, se realiza sobre la base de los instrumentos validados y utilizados en el estudio, que nos brinda información pertinente de las variables más relevantes asociadas al problema de investigación, permitiendo de esta manera realizar inferencias válidas para el estudio. En primera instancia se realiza un análisis descriptivo de las variables más relevantes del estudio, para luego realizar el análisis estadístico correlacional para la comprobación de la hipótesis. Se aplicó los instrumentos de recolección de datos a 156 estudiantes de la Institución
"Santiago León" del Distrito de Chongos BajoChupaca, mediante un análisis de las tres pruebas de correlación (Coeficiente de Correlación " $r$ " de Pearson, Coeficiente de Correlación Tau b Kendall y el coeficiente de correlación Rho de Spearman). Por último se realizó un análisis descriptivo de cada variable. En la prueba de hipótesis General se utilizó el Coeficiente de "r" de Pearson por tratarse de variables cuantitativas.

Educativa "Santiago León" del Distrito de Chongos Bajo, los cuales ingresaron al estudio previo consentimiento informado.

Depresión en los adolescentes de la institución educativa "Santiago León" del distrito de Chongos Bajo:

Los puntajes de la aplicación de la escala permite evaluar los niveles de depresión en los adolescentes de la Institución Educativa "Santiago león" del Distrito de Chongos Bajo se detallan en los siguientes gráficos.

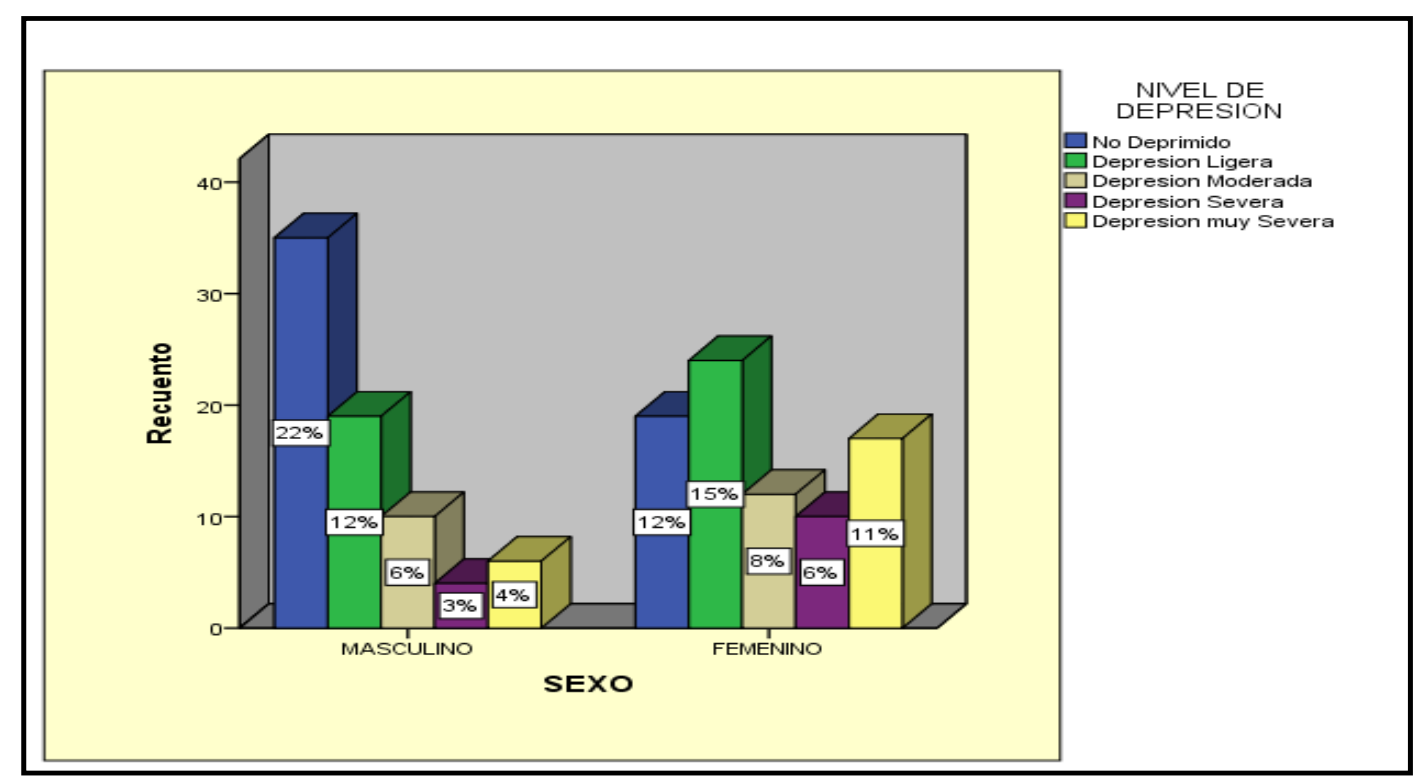

\section{Grafico 1: Nivel de depresión según el sexo de los adolescentes de la institución educativa "Santiago León" del distrito de Chongos Bajo- 2014.}

Fuente: Datos recopilados por los investigadores-Chongos Bajo-2014. 


\section{Prueba de hipótesis:}

Se debe demostrar que las frecuencias de los cinco Niveles de Depresión no son homogéneos, es decir que existe una mayor cantidad de adolescentes en uno de los cinco Niveles de Depresión, específicamente en el Nivel de Depresión Ligera, para ello planteamos la siguiente hipótesis:

\section{Prueba de hipótesis específica $\mathrm{N}^{\mathrm{a}} \mathbf{1}$ :}

Existe un nivel de depresión ligera en los adolescentes de la Institución Educativa "Santiago León" del Distrito de Chongos Bajo-Chupaca 2014.

La prueba de hipótesis adecuada es la prueba de bondad de ajuste de Chi-cuadrado con ello se formula la siguiente Hipótesis Nula $\left(\mathrm{H}_{0}\right)$ y la Hipótesis Alterna $\left(\mathrm{H}_{1}\right)$.

$\left(\mathbf{H}_{\mathbf{0}}\right)$ : No existe diferencia en los niveles de depresión en los adolescentes de la Institución Educativa "Santiago León" del Distrito de Chongos Bajo.
H1: Existe diferencia en los niveles de depresión en Los adolescentes de la Institución Educativa "Santiago León" del Distrito de Chongos Bajo

Según la regla de decisión: Se rechaza la $\mathbf{H}_{\mathbf{0}}$ si ocurre que:

$$
\begin{array}{r}
\mathrm{x}^{2} \text { calculada }>\mathrm{x}^{2} \text { térico } \\
35.47>\mathbf{9 . 4 8} \ldots . .
\end{array}
$$

Como la inecuación es verdadera con un nivel de significancia de 0.05 y con cuatro grados de libertad se rechaza la Hipótesis Nula y se acepta la Hipótesis Alterna es decir que: Existe diferencia en los Niveles de Depresión en los adolescentes de la Institución Educativa "Santiago León" del Distrito de Chongos Bajo. Al existir diferencia entre los niveles, se afirma con un nivel de confianza 1$\alpha=0,95$ que: Existe un Nivel de Depresión Ligera en los adolescentes de la Institución Educativa "Santiago León" del Distrito de Chongos BajoChupaca 2014.

Ideación suicida en los adolescentes de la institución educativa "Santiago León" del distrito de Chongos Bajo.

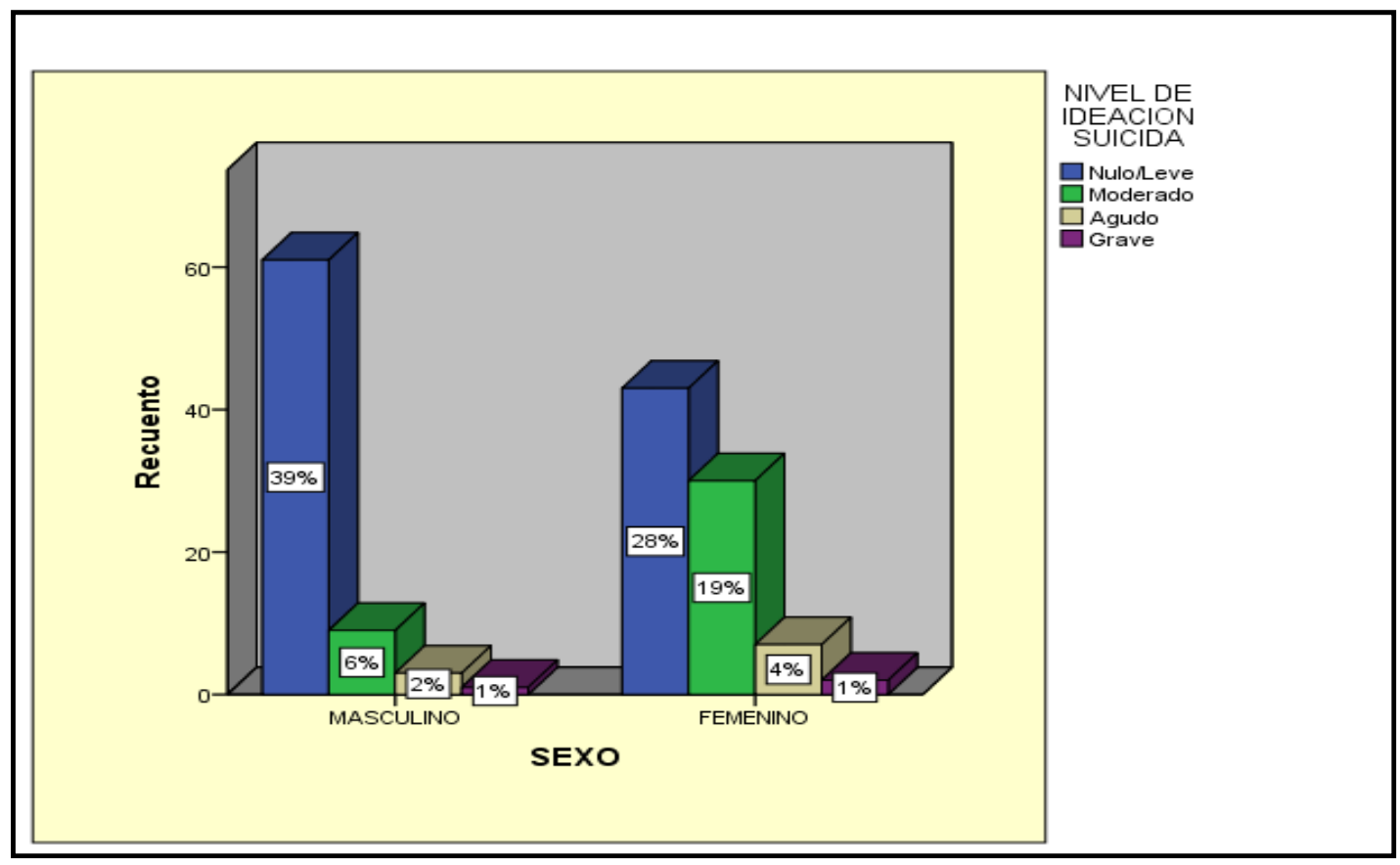

Grafico 2: Nivel de ideación suicida según el sexo de los adolescentes de la institución educativa "Santiago León" del distrito de Chongos Bajo-2014.

Fuente: Datos recopilados por los investigadores-Chongos Bajo-2014 
En el Grafico 2, se muestra que 67\% (104/156) adolescentes encuestados de la Institución Educativa "Santiago León" del Distrito de Chongos Bajo (39\% de sexo Masculino y 28\% de sexo Femenino) presentan Ideación Suicida Nulo/Leve; mientras que el 33\% (52/156) adolescentes (9\% de sexo Masculino y 24\% de sexo Femenino) si presentan algún tipo de Ideación Suicida. Según el Sistema Nacional de Salud existen patrones de suicidio en cuanto al sexo, pero no son iguales en todos los países. En general el suicidio es más común en varones, pero las mujeres realizan más intentos de suicidio. (41)

\section{Prueba de hipótesis:}

Se debe demostrar que las frecuencias de los cuatro Niveles de Ideación Suicida no son homogéneos, es decir que existe una mayor cantidad de adolescentes en uno de los cuatro Niveles de Depresión, específicamente en el Nivel Moderado de la Ideación Suicida, para ello planteamos la siguiente hipótesis:

\section{Prueba de hipótesis específica $\mathrm{n}^{0}$ 2:}

Existe un nivel de ideación suicida moderado en los adolescentes de la Institución Educativa "Santiago León" del Distrito de Chongos Bajo-Chupaca 2014.

La prueba de hipótesis adecuada es la prueba de bondad de ajuste de Chi-cuadrado con ello se formula la siguiente Hipótesis Nula $\left(\mathrm{H}_{0}\right)$ y la Hipótesis Alterna $\left(\mathrm{H}_{2}\right)$.

$\left(\mathbf{H}_{\mathbf{0}}\right)$ : No existe diferencia en los niveles de ideación suicida en los adolescentes de la Institución Educativa "Santiago León" del Distrito de Chongos Bajo.

H2: Existe diferencia en los niveles de ideación suicida en Los adolescentes de la Institución Educativa "Santiago León" del Distrito de Chongos Bajo.

Según la regla de decisión: Se rechaza la $\mathbf{H}_{0}$ si ocurre que:

$$
\mathrm{X}^{2} \text { calculada }>\mathrm{x}^{2} \text { teórico }
$$

$163.13>7.85$

Como se observa en la inecuación es verdadera con un nivel de significancia de 0.05 y con tres grados de libertad se rechaza la Hipótesis Nula y se acepta la Hipótesis Alterna es decir que: Existe diferencia en los niveles de Ideación Suicida en los adolescentes de la Institución Educativa "Santiago León" del Distrito de Chongos Bajo. Al existir diferencia entre los niveles, se afirma con un nivel de confianza 1$\alpha=0,95$ que: Existe un Nivel de Ideación Suicida Moderado en los adolescentes de la Institución Educativa "Santiago León" del Distrito de Chongos Bajo-Chupaca 2014.

\section{Análisis de correlación de los niveles de depresión e ideación suicida:}

Mediante el software del paquete estadístico SPSS versión 21 se procedió a analizar el coeficiente de correlación de los puntajes obtenidos de la Escala de Depresión e Ideación Suicida en los adolescentes de la Institución Educativa "Santiago León" del Distrito de Chongos Bajo, mediante tres pruebas de correlaciones: Coeficiente de correlación " $r$ " de Pearson, el coeficiente de Tau b Kendall y el coeficiente de Rho de Spearman los resultados son los siguientes:

La tabla 1, de correlaciones nos muestra que los diferentes Niveles de Depresión de los adolescentes y los diferentes Niveles de Ideación Suicida de los adolescentes están relacionados, así también en general Depresión e Ideación Suicida están relacionados en los adolescentes encuestados de la Institución Educativa "Santiago León" del Distrito de Chongos Bajo en el año 2014, siendo su coeficiente de correlación 0,560 significativa al nivel 0,01 (2 Colas/Bilateral), sin embargo el que tiene la mayor fuerza de correlación, es el Nivel de Depresión Muy Severa con el Nivel de Ideación Suicida Grave, siendo su coeficiente de correlación 0,829 significativa al nivel 0,01 (2 Colas/Bilateral). 
Tabla l: CORRELACIÓN ENTRE EL NIVEL DE DEPRESIÓNE IDEACIÓN SUICIDA EN LOS ADOLESCENTES DE LA INSTITUCIÓN EDUCATIVA "SANTIAGOLEÓN" DEL DISTRITO DE CHONGOS BAJO- 2014.

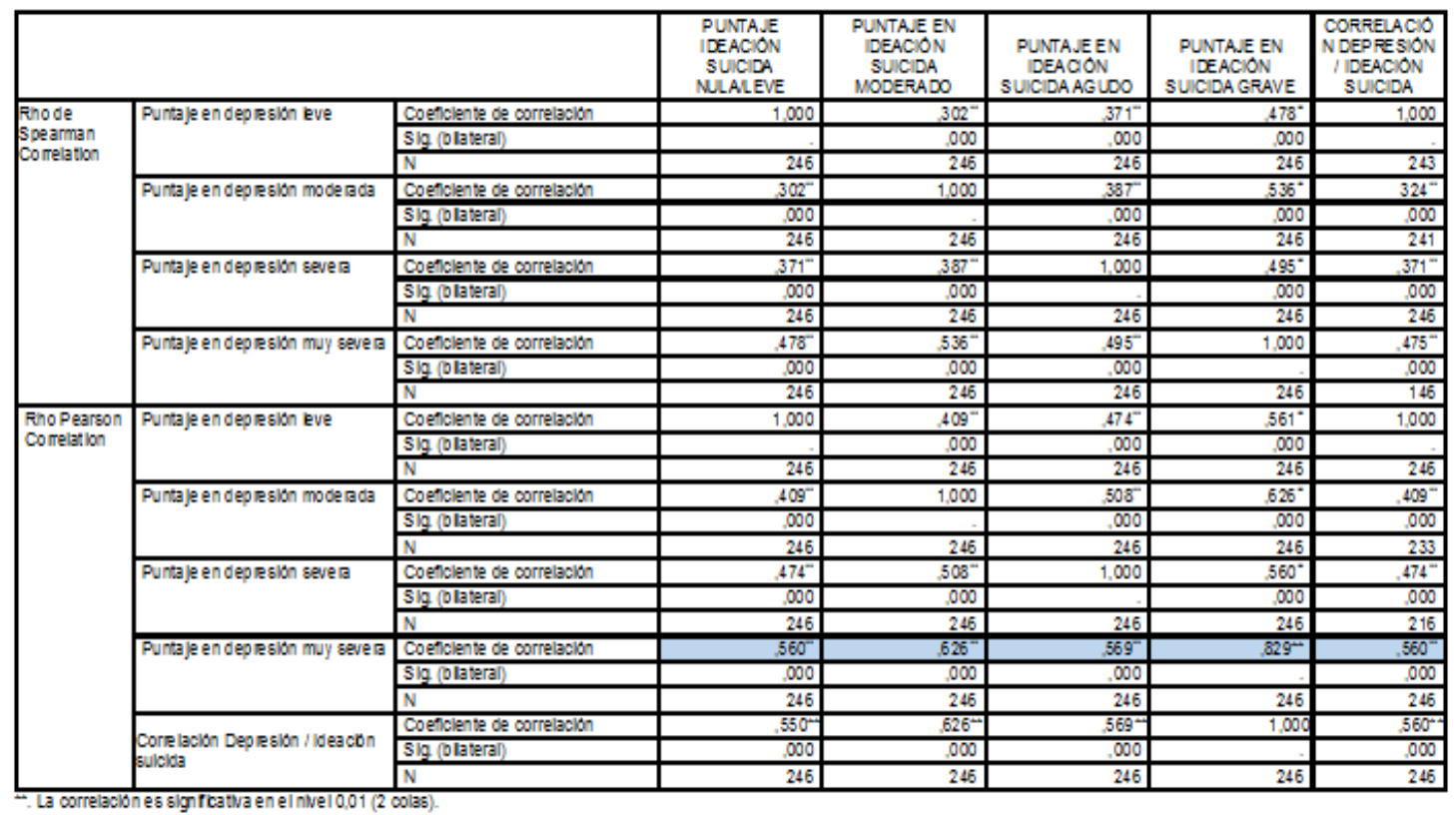

\section{Prueba de hipótesis general:}

En la presente investigación se formuló la siguiente hipótesis:

Existe una relación significativa entre Depresión e Ideación Suicida en los adolescentes de la Institución Educativa "Santiago León" del Distrito de Chongos Bajo.

Es decir:

A mayor Nivel de Depresión existe una mayor presencia de Ideación Suicida en los adolescentes de la Institución Educativa "Santiago León” del Distrito de Chongos Bajo.

A menor Nivel de Depresión existe menor presencia de Ideación Suicida en los adolescentes de la Institución Educativa "Santiago León" del Distrito de Chongos Bajo.

Las hipótesis a contrastar:

$\left(\mathbf{H}_{\mathbf{0}}\right)$ : No existe relación significativa entre Depresión e Ideación Suicida en los adolescentes de la Institución Educativa "Santiago León" del Distrito de Chongos Bajo-Chupaca 2014.

$\left(\mathbf{H}_{\mathrm{I}}\right)$ : Existe una relación significativa entre Depresión e Ideación Suicida en los adolescentes de la Institución Educativa "Santiago León" del Distrito de Chongos Bajo-Chupaca 2014.

\section{R DE PEARSON: \\ NIVEL DE SIGNIFICANCIA Y GRADOS DE LIBERTAD:}

En ésta investigación se realizó la prueba de hipótesis con un nivel de significancia de $0.05 \mathrm{y}$ de grados de libertad 154. (n-2)

- Nivel de significancia $(\alpha)$

$\alpha=0.05 \quad \mathrm{gl}=150$ entonces $\mathrm{r}$ $\alpha=0.159 \ldots \ldots . \mathrm{gl}=175$ entonces $\mathrm{r} \alpha=0.148$

Donde gl (154) se ubica entre $\mathrm{r} \alpha=0.159 \mathrm{r} \alpha=0.148$ que se tomó de la tabla de correlación de Pearson, a 150 y 175 grados de libertad respectivamente y 0.05 de nivel de significancia.

Se calcula el coeficiente de Pearson calculado entre los puntajes de la Escala de Depresión y el puntaje de la Escala de Ideación Suicida en los adolescentes de la Institución Educativa "Santiago León" del Distrito del Distrito de Chongos Bajo donde :

$\mathrm{r}_{\mathrm{c}}=0.560$

Regla de decisión: Se rechaza la H0 si ocurre que:

$\mathrm{rc}>\mathrm{r} \alpha$

$0.560>(0.159$ entre 0.175$) \ldots \ldots . . . . . . . .(v)$

Mediante un análisis paramétrico de " $R$ " de Pearson calculada de $\mathrm{rc}=0.560$ con un nivel de 
significancia de $\alpha=0,05$ y grados de libertad $(\mathrm{gl})=154$ se rechaza la Hipótesis Nula $(\mathrm{H} 0)$ y por ende se acepta la Hipótesis de Investigación (HI) Entonces decimos que: Existe una relación significativa entre los puntajes de la Escala de Depresión y la Escala de Ideación Suicida en los adolescentes de la Institución Educativa "Santiago León” del Distrito de Chongos Bajo.

\section{DISCUSIÓN}

Términos como Depresión o Ideación Suicida parecieran estar muy alejados de los adolescentes. Sin embargo, hoy en día existen pocas dudas acerca de la presencia de estas variables Depresión e Ideación Suicida en los adolescentes, que experimentan circunstancias personales difíciles de superar. En la revisión del estado de la cuestión, la Ideación Suicida aparece como una variable que puede determinar niveles patológicos en cuanto a los pensamientos y deseos más profundos del adolescente sobre la propia muerte. Durante la adolescencia suelen ser frecuentes este tipo de pensamientos y para algunos jóvenes esa etapa no se recorre como un periodo apacible, sino como un periodo de intensas vivencias donde aparecen sentimientos y actitudes contradictorias en los diferentes ámbitos de su vida. Esas vivencias comprenden, desde la confrontación de la propia identidad personal, hasta ámbitos más externos como la relación con los iguales y con la sociedad adulta.

En investigaciones actuales en Europa y Estados Unidos las cifras de Ideación Suicida oscilan entre el $5 \%$ y el $27 \%$ (3). En algunas ocasiones, la conducta que acompaña la Ideación Suicida es el Suicidio consumado, que hoy aparece como una de las primeras causas de muerte en la adolescencia. En el Reino Unido y Francia, por ejemplo, es la Segunda Causa de muerte entre los sujetos de 15-24 años después de los accidentes de tráfico. En España aproximadamente unos 1000 jóvenes con edades entre 15 y 24 años se suicidan anualmente siendo más frecuente en los chicos (4). Las tasas de suicidio se han triplicado en los últimos años, mientras que las tentativas se mueven entre el $9 \%$ y el $11 \%$ en la franja de edad anteriormente citada. Para la OMS, el Suicidio es la causa de la mitad de todas las muertes violentas y se traduce en casi un millón de víctimas al año; la misma organización estima que en el año 2020 las víctimas podrían ascender a 1.5 millones. $\mathrm{La}$ adolescencia es considerada una etapa del desarrollo caracterizada por continuos cambios, que pueden ocasionar estrés. Las exigencias
Entonces se demuestra que: La Depresión y la presencia de la Ideación Suicida en los adolescentes de la Institución Educativa "Santiago León" del Distrito de Chongos Bajo se relacionan significativamente,medianamente y positivamente.

psicosociales derivadas de este proceso evolutivo pueden repercutir en el desarrollo psicológico, influyendo en la confianza en sí mismo, la timidez, la ansiedad, el sentimiento de autoeficacia, la autoestima o el desarrollo de estrategias de afrontamiento. Todo esto implica que la población adolescente presenta un alto riesgo hacia la Depresión e Ideación Suicida. En el Perú, existe muy escasa información y pocos estudios respecto al problema en población escolar. Al respecto, el Instituto Nacional de Salud Mental "Honorio Delgado - Hideyo Noguchi” realizó una encuesta de Salud Mental a nivel de población general de Lima Metropolitana (4). En una muestra probabilística de 991 adolescentes en el rango de 12 a 17 años encontraron las siguientes prevalencias de vida: $29.1 \%$ de "Consideraciones y pensamientos suicidas" y $3.6 \%$ de Intento Suicida. Por otro lado, precisemos que la conducta suicida suele vincularse a diversos factores de riesgo entre los cuales se señalan el antecedente de un intento previo, depresión, abuso de alcohol, alteraciones de la personalidad y otros trastornos psiquiátricos y habilidades para la vida. (5)

En el marco de lo explicitado, los resultados del presente estudio muestran que Depresión e Ideación Suicida están relacionados en los Adolescentes encuestados de la Institución Educativa "Santiago León" del Distrito de Chongos Bajo, siendo su coeficiente de correlación 0,560 significativa al nivel 0,01 Colas/Bilateral); los resultados de nuestro estudio concuerdan con los hallazgos de Coffin N. Álvarez M. Marin M. (6), Perales A. et al. (8), quienes encontraron una correlación estadísticamente significativa entre Depresión e Ideación Suicida, estableciendo al mismo tiempo que los Niveles de Ideación Suicida encontrados (Moderado y Alto), son también un factor de riesgo para desarrollar niveles graves de la misma. Nuestro reporte también coincide con los reportes de Juracán M. et al (7); Ailee B. Minetto A. et al (10); Cano, P. Gutiérrez, C. Nizama, M. (9); Hinostroza L. Huashuayo, C. Navarro S. Torres, C. Matos L. (10); quienes hallaron estudiando por separado el factor 
Depresión y el factor Ideación Suicida, que ambas variables se correlacionan significativamente en estudiantes de Nivel Secundario, en adolescentes y en estudiantes de Pregrado de Universidades Públicas, productos que concuerdan con los resultados obtenidos en el presente estudio.

Por otro lado Perales A. et al. (8) en el estudio sobre "Conducta suicida en estudiantes de las escuelas de educación de la Universidad Nacional Mayor de San Marcos", en el análisis bivariado realizado, el intento suicida se asoció significativamente a niveles altos de Depresión $\mathrm{OR}=6,05, \mathrm{p}=0,001$; de Ansiedad $\mathrm{OR}=7,12, \mathrm{p}=$ 0,001; Pensamiento Homicida $\mathrm{OR}=3,15, \mathrm{p}=0,001$; indicadores de Conducta Antisocial $\mathrm{OR}=1,93$, $\mathrm{p}=0,02$; y Riesgo de Problemas con el Alcohol (CAGE positivo) $\mathrm{OR}=3,11, \quad \mathrm{p}=0,007$. Concluyendo que la población estudiantil de las Escuelas de Educación de la UNMSM constituye un grupo en riesgo en cuanto a indicadores suicidas asociados a otras variables de Salud Mental como es la Depresión. De igual forma Rosas M.; Yampufé M.; Milena López M.; Carlos G.; Sotil A. (9), en otro estudio sobre "Niveles de Depresión en estudiantes de Tecnología Médica", muestra que los alumnos de la EAP Tecnología Médica, entre 20 y 25 años, una de cada dos presentó algún nivel de Depresión. Se extrapola que, en el caso de los estudiantes varones de la EAP Tecnología Médica, 56 de cada 100 presentan algún nivel de Depresión, por lo que se recomienda realizar programas de Prevención de Depresión y determinar las causas subyacentes, hallazgos que también concuerdan con los resultados del presente estudio.

En este contexto en los últimos años en las Instituciones Educativas se ha visto con mucha preocupación, un incremento en el Comportamiento Suicida de la población escolar de Nivel Secundaria, sin embargo no existen reportes válidos y confiables de la magnitud del problema y su relación con otros factores como es la Depresión en escolares en la Región Junín, por tanto los resultados del presente estudio, aparte de llenar un vacío importante de información científica sobre el tema, deben ser utilizados para la organización de un Programa Preventivo de la Depresión e Ideación Suicida y de intervención en favor de los estudiantes de esta Institución Educativa, siendo una responsabilidad moral y científica de ocuparse no sólo de instruir a sus estudiantes sino de velar por su formación y desarrollo integral, como personas.

\section{CONCLUSION}

Los adolescentes encuestados de la Institución Educativa "Santiago León" del Distrito de Chongos Bajo en el año 2014, el 65\% presentan algún tipo de Depresión. Es así que en esta Investigación se ha mostrado que la Depresión en la adolescencia a menudo persiste, recurre y se prolonga en la adultez especialmente si no se recibe tratamiento.

Los adolescentes encuestados de la Institución Educativa "Santiago León" del Distrito de Chongos Bajo en el año 2014, el 33\% presentan algún tipo de Ideación Suicida. Es así que la problemática de suicidio en la población adolescente es un tópico relativamente reciente, pero que amerita atención urgente; pues las condiciones que la subyace también tienden al aumento y con ello, la tendencia al riesgo suicida es cada vez mayor.

Depresión e Ideación Suicida, están relacionados en los adolescentes encuestados de la Institución Educativa "Santiago León" del Distrito de Chongos Bajo en el año 2014, siendo su coeficiente de correlación 0,560 significativa al nivel 0,01 (2 Colas/Bilateral).

El Nivel de Depresión Muy Severa y el Nivel de Ideación Suicida Grave, tienen la mayor fuerza de correlación, siendo su coeficiente de correlación 0,829 significativa al nivel 0,01 (2 Colas/Bilateral).

Es necesario, importante e ineludible la implementación de programas preventivos de la Depresión e Ideación Suicida y de intervención en favor de los estudiantes de esta Institución Educativa y de otros escenarios Socio geográficos similares, siendo una responsabilidad moral y científica de ocuparse no sólo de instruir a sus estudiantes sino de velar por su formación $\mathrm{y}$ desarrollo integral, como personas.

\section{AUTOR DE CORRESPONDENCIA}

Walter Edgar Gómez Gonzales Universidad Norbert Wiener - UNW. Avenida Arequipa 440-Santa Beatriz-Lima Teléfono: 01-706-5100

E-mail: waltergomez29@yahoo.com 


\section{REFERENCIAS BIBLIOGRAFICAS}

1. OMS. Depresión. [En línea]; 2012[Fecha de acceso 15 de Setiembre del 2014]. URL:

http://www.who.int/mediacentre/factsheet s/fs369/es/.

2. OMS, En el 2020 la Depresión será la segunda causa de incapacidad en el mundo. [En línea].; 2007[Fecha de acceso 15 de setiembre del 2014]. URL:http://www.psiquiatria.com/depresi on/segun-la-oms-en-el-2020-ladepresion-sera-la-segunda-causa-deincapacidad-en-el-mundo/.

3. Buendía, J., Riquelme, A. y Ruíz, J.A. El suicidio en adolescentes. Factores implicados en el comportamiento suicida. 2004. Edita: Universidad de Murcia.

4. Instituto Especializado de Salud Mental. Encuesta de Salud mental de Lima Metropolitana. Anales de Salud Mental, 2002: 1-197.

5. Kijtorntham, Wichuda. The Causal Relationship Model of Life Skills and Risk Behavior of Undergraduate Students in Thailand false Asian Social Science 9.1 (Jan 2013): 105-111.

6. Coffin N. Álvarez M. Marin M. Depresión e ideación suicida en estudiantes de la FESI: Un estudio piloto: Revista Electrónica de Psicología Iztacala: 2011; 14(4):341-354.

7. Juracán M. Sintomatología de ansiedad, depresión e ideación suicida en pacientes con acné. [Tesis bachillerato]. GUATEMALA: 2010.

8. Perales A. et al. Conducta suicida en estudiantes de las escuelas de educación de la Universidad Nacional Mayor de San
Marcos. Investigación Educativa Vol. 17, N. ${ }^{\circ}$ 2, 185-194 Julio-Diciembre 2013, ISSN 1728-5852.

9. Rosas M.; Yampufé M.; Milena López M.; Carlos G.; Sotil A. Niveles de depresión en estudiantes de Tecnología Médica. Revista An Fac med. 2011;72(3):183-6

10. Ailee B., MinettoA. Violencia familiar e ideación suicida en adolescentes del Hospital San Juan Bautista de Huaral [Tesis licenciatura]. Lima-Perú: Universidad César Vallejo;2013.

11. CANO, Pablo; GUTIERREZ, César y NIZAMA, Martín. Tendencia a la violencia e ideación suicida en adolescentes escolares en una ciudad de la Amazonia Peruana. Rev. Perú. med. exp. Salud Pública [online]. 2009, vol.26, n.2 [citado 2015-02-17], pp. 175-181.

12. Hinostroza G. Huashuayo C. Navarro S. Dinámica familiar y manifestaciones de depresión en escolares de 13 a 15 años de edad: Rev enferm Herediana:2011;4(1):12-19.

13. Buendía, J., Riquelme, A. y Ruíz, J.A. El suicidio en adolescentes. Factores implicados en el comportamiento suicida. 2004. Edita: Universidad de Murcia.

14. Instituto Especializado de Salud Mental. Encuesta de Salud mental de Lima Metropolitana. Anales de Salud Mental, 2002: 1-197.

15. Kijtorntham, Wichuda. The Causal Relationship Model of Life Skills and Risk Behavior of Undergraduate Students in Thailand false Asian Social Science 9.1 (Jan 2013): 105-111.

Recibido: 28/01/2016

Aceptado: 29/04/2016 